Document downloaded from:

http://hdl.handle.net/10251/34829

This paper must be cited as:

Fogue, M.; Garrido, P.; Martínez, FJ.; Cano Escribá, JC.; Calafate, CT.; Manzoni, P. (2013). A novel approach for traffic accidents sanitary resource allocation based on multiobjective genetic algorithms. Expert Systems with Applications. 40(1):323-336. doi:10.1016/j.eswa.2012.07.056.

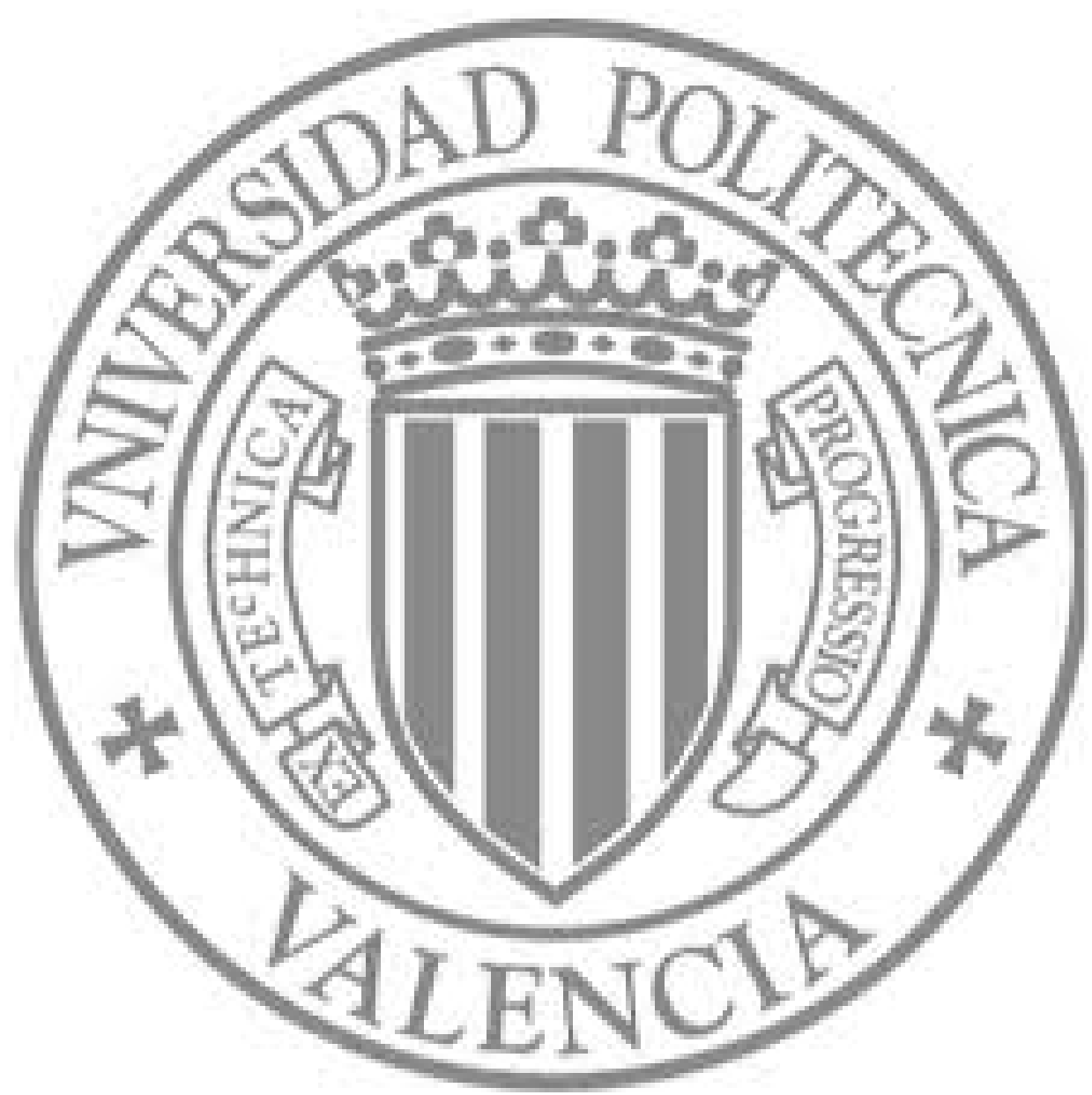

The final publication is available at

http://dx.doi.org/10.1016/j.eswa.2012.07.056

Copyright Elsevier 


\title{
A Novel Approach for Traffic Accidents Sanitary Resource Allocation Based on Multi-Objective Genetic Algorithms
}

\author{
Manuel Fogue, Piedad Garrido, Francisco J. Martinez \\ University of Zaragoza, Spain \\ Email: \{mfogue, piedad, f.martinez\}@unizar.es \\ Juan-Carlos Cano, Carlos T. Calafate, Pietro Manzoni \\ Universitat Politècnica de València, Spain \\ Email: \{jucano, calafate, pmanzoni\}@disca.upv.es
}

\begin{abstract}
The development of communication technologies integrated in vehicles allows creating new protocols and applications to improve assistance in traffic accidents. Combining this technology with intelligent systems will permit to automate most of the decisions needed to generate the appropriate sanitary resource sets, thereby reducing the time from the occurrence of the accident to the stabilization and hospitalization of the injured passengers. However, generating the optimal allocation of sanitary resources is not an easy task, since there are several objectives that are mutually exclusive, such as assistance improvement, cost reduction, and balanced resource usage. In this paper, we propose a novel approach for the sanitary resources allocation in traffic accidents. Our approach is based on the use of multi-objective genetic algorithms, and it is able to generate a list of optimal solutions accounting for the most representative factors. The inputs to our model are: i) the accident notification, which is obtained through vehicular communication systems, and ii) the severity estimation for the accident, achieved through data mining. We evaluate our approach under a set of vehicular scenarios, and the results show that a memetic version of the NSGA-II algorithm was the most effective method at locating the optimal resource set, while maintaining enough variability in the solutions to allow
\end{abstract}


applying different resource allocation policies.

Keywords: Resource Allocation; Traffic Accidents Assistance; Multi-objective Genetic Algorithms

\section{Introduction}

During the last decades, traffic safety has become crucial in most countries around the world. The growth of the number of vehicles leads to more dangerous roads, which requires drivers to have higher levels of attention. This situation has dramatically increased the amount of traffic accidents, producing 2,478 fatalities in Spanish roads during 2010, which means one death for every 18,551 inhabitants (Dirección General de Tráfico (DGT), 2010); additionally, 34,500 people died in the whole European Union as a result of a traffic accident in 2009 (Eurostat: Statistical Office of the European Communities, 2012).

To reduce the number of road fatalities, vehicular networks will play an increasing role in the Intelligent Transportation Systems (ITS) area (Figueiredo et al., 2001). Most ITS applications, such as road safety, fleet management, and navigation, will rely on data exchanged between vehicles and the roadside infrastructure (V2I), or even directly between vehicles (V2V) (Miller, 2008). The integration of sensoring capabilities on-board vehicles, along with peer-topeer mobile communication among vehicles, forecast significant improvements in terms of safety in the near future.

In this scenario, the elapsed time from the accident occurrence to the moment where the affected passengers are stabilized and hospitalized is critical to increase their survival probability, while reducing the severity of their injuries. This concept is commonly known as the Golden Hour (Martinez et al., 2010). ITS services and communication technologies may definitely help at reducing this time by providing fast accident notification to the control centers in charge of traffic surveillance; however, those services still demand an accurate estimation of the severity of the accident, and the potential danger for the occupants to generate an adequate emergency response to assist the injured. Therefore, 
communication between vehicles should be supported by an infrastructure providing intelligent systems, capable of automatically deploying the set of actions required for each specific accident.

A preliminary assessment of the accident severity will help emergency services to adapt the human and material resources to the conditions of the accident, with the consequent assistance quality improvement (Fogue et al., 2011, 2012a). Data mining and vehicular networks can be successfully used together to notify and make a preliminary estimation of the severity of the accident, both in the injuries produced on the passengers and the damages on the vehicles (Fogue et al., 2012b). However, it is still necessary to define how this information can be used to automatically achieve optimal resource allocation for the emergency operatives assigned to a crash. In this paper, we propose a novel approach based on i) the information collected by vehicular networks, and ii) the severity estimations provided by data mining. Our solution provides i) the most adequate set of resources for a specific traffic accident scenario, and ii) information about which suppliers should provide these resources depending on factors like their proximity to the affected area, available resources, and experience dealing with similar accident situations. Our proposal is able to increase the chances of survival for the affected people, focusing on improving post-collision assistance.

The rest of the paper is organized as follows: Section 2 presents a sanitary resource classification for traffic accidents. Section 3 shows the basis of the Multi-objective Optimization problems and how Genetic Algorithms (GA) can solve these problems. Section 4 presents our proposal for resource allocation. Section 5 evaluates the obtained results using a set of traffic accident scenarios. Finally, Section 6 concludes this paper.

\section{Sanitary resources required in a traffic accident}

In every traffic accident involving injured people, it is essential to provide health care as soon as possible to minimize the potential damage to the occupants of the affected vehicles. However, not all the available ambulances and 
rescue teams incorporate the same equipment and medical staff, meaning that there are different types of vehicles with different medical attention capabilities (Spanish Ministry of Health, 1998).

A preliminary classification of sanitary vehicles divides them into assistance and non-assistance vehicles. The former allow providing health care during the transport of the patients, including the necessary sanitary equipment and staff, whereas the latter are merely able to transport patients on stretchers, being not specifically equipped for medical care. However, a closer look to the different types of assistance ambulances shows that, depending on the severity of injuries they are prepared to handle, they can be classified in the following groups (see Figure 1):

- Non-assistance Ambulances: also known as transport-only or support ambulances, consist of vehicles designed for the evacuation of patients alone, thereby not being specially equipped for providing assistance. The staff usually consists of one or two technicians, and the equipment is very basic, being unsuitable for the transportation of urgent patients. They usually include a stretcher and two seats in the rear area of the ambulance.

- Basic Life Support (BLS) Assistance Ambulances: vehicles equipped with all the equipment required to provide basic life support to the patient, reducing the risk of death or other consequences resulting from the injury or from the transport conditions. They are known as basic ambulances, or just BLS. The staff includes two trained technicians equipped with the basic equipment for the patient assistance and stabilization, i.e., material for first aid and immobilization, and oxygen. They can be used for first aid, as well as for the treatment and transportation of patients with minor injuries.

- Advanced Life Support (ALS) or Mobile Intensive Care Ambulances: vehicles with elements able to provide advanced life support, and the practice of surgery to the patient. The staff includes a doctor, a nurse, and at 
least one trained driver. In addition to the material of BLS ambulances, they include medication and electromedical equipment, providing all the needed material to treat patients with serious injuries while being carried to a hospital.

- Fast Intervention Vehicles (FIV): also known as medical vehicles, they are usually SUVs or large cars equipped with sanitary equipment to attend areas with rough terrain, under adverse weather conditions or in cases where special services are carried. The staff includes a a doctor and/or a nurse and a paramedic, and the available equipment is the same as for ALS units; however, they are unable to move patients, since they do not include stretchers. They are useful for their agility and speed, but they require an extensive network of BLS ambulances to provide transportation capabilities.

- Health Emergency Helicopters (HEH): air vehicles especially useful for reaching areas far away from hospitals. The staff includes a pilot, a mechanic, a doctor, a nurse, and sometimes a medical technician. The equipment is the same as for ALS ambulances, but they may not be used in urban areas due to the difficulty of finding adequate landing areas, and they require the existence of heliports at the final destination hospitals.

\subsection{Features of the different sanitary vehicles}

The existing sanitary vehicles used to provide support for traffic accidents can be classified according to several factors, related to the type of injured passengers they are able to assist, their ability to reach the crash site, and so on.

\subsubsection{Severity of injuries supported}

The injury severity or assistance category indicates the type of injuries for which the vehicle includes medical supplies and human resources. This assistance category is divided into three levels: 


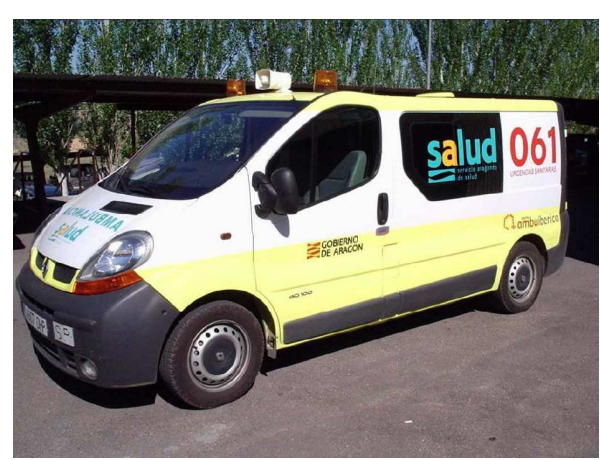

(a)

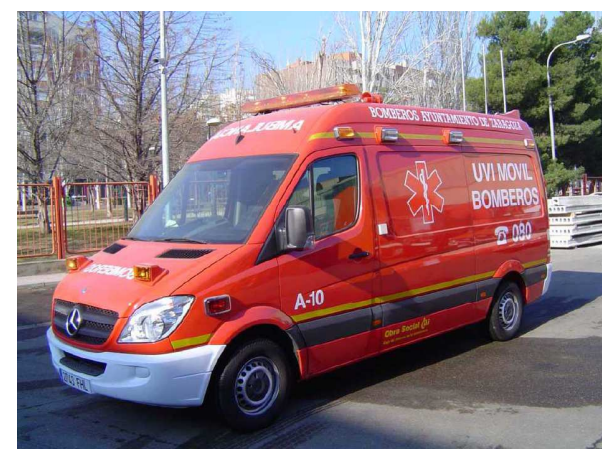

(c)

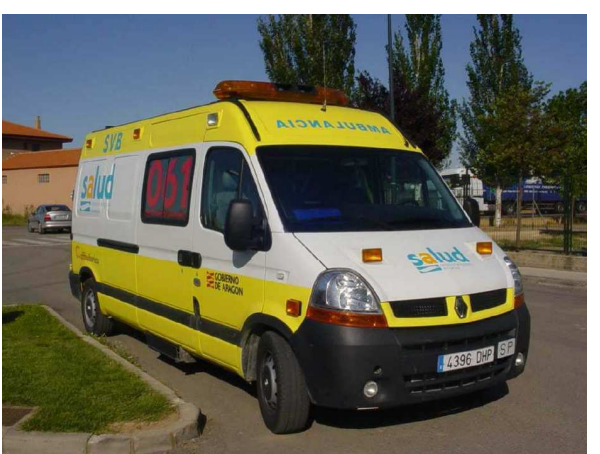

(b)

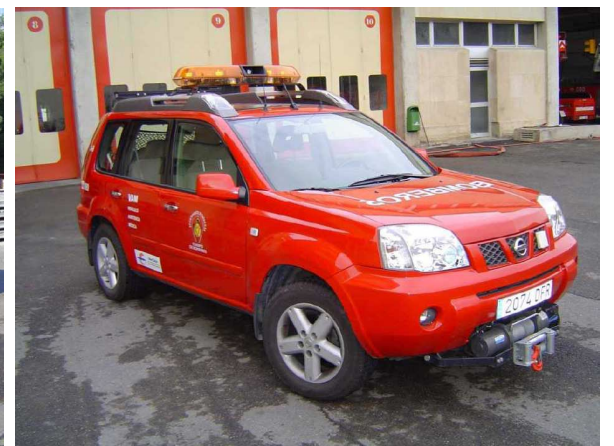

(d)

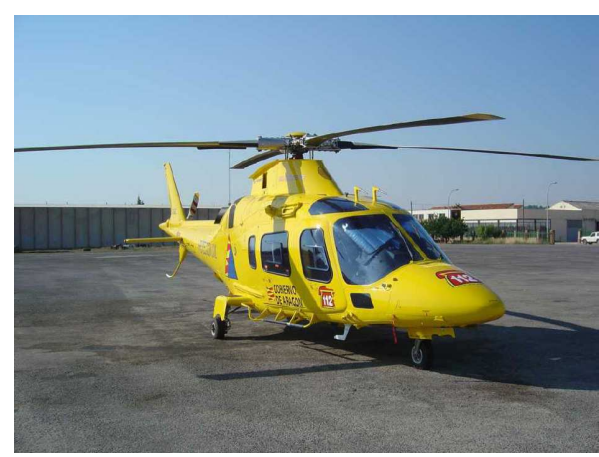

(e)

Figure 1: Classification of sanitary vehicles needed in a traffic accident: (a) Non-assistance ambulance, (b) BLS ambulance, (c) ALS ambulance, (d) FIV vehicle, and (e) HEH helicopter. 
- Non-assistance: the vehicle does not include enough material to heal any type of wound that can not be stabilized by first aid. This type of vehicle can only be used for the transportation of passengers without serious injuries, and when the transportation time is not especially important. Non-assistance ambulances belong to this category.

- Basic Life Support (BLS): the vehicle contains additional material to stabilize the patient (immobilization equipment and oxygen), so it is possible to transport passengers with moderately severe injuries, or those which can be stabilized on the way to the hospital. BLS ambulances belong to this category.

- Advanced life support (ALS): the vehicle incorporates intensive care material and allows practicing surgery for severely injured patients. This type of transportation is particularly suitable for occupants who have suffered a severe accident, and so a fast intervention is crucial to ensure their survival. This category includes ALS ambulances, FIV vehicles, and HEH helicopters.

\subsubsection{Passenger capacity}

The capacity of a vehicle is expressed by the maximum number of passenger that can be transported in a standard service (depending on the seriousness of injuries). Passengers with severe injuries should be evacuated individually in different vehicles, due to the difficulty to stabilize them.

The maximum passenger capacity for the different medical vehicles is usually:

- Non-assistance Ambulance: 1 or 2 occupants

- BLS Ambulance: 1 or 2 occupants

- ALS Ambulance: 1 occupant

- Fast Intervention Vehicle (FIV): 0 occupants

- Helicopter (HEH): 1 occupant 
Vehicles in charge of evacuating people with minor injuries would be able to carry more than one person at once, if they have been previously stabilized. FIV vehicles can not transport any patients. Finally, both ALS ambulances as HEMS helicopters are capable of transporting seriously injured passengers.

\subsubsection{Accessible areas}

According to the characteristics of the vehicles, there are areas where access may be more or less complicated, or that directly impede their use in that area. The different zones can be classified as urban (cities or environments with a high density of buildings), interurban (roads and highways between cities or small towns with few buildings), and rough terrain (difficult road conditions and mountain area, among others). If an accident happens in an area with rough terrain, vehicles will find difficult reaching the accident area, and therefore their speed may be considerably reduced. Ambulances should generally move more slowly to reach an area of difficult access, whereas FIV vehicles are less affected and helicopters are not affected at all by the terrain. However, helicopters are not usually able to land with enough security in urban areas with many buildings.

\subsubsection{Average speed}

Each vehicle presents different speed depending on the area where it is moving, mostly due to the road conditions or the traffic laws. Our approach will consider the average speeds for the sanitary vehicles shown in Table 1.

\subsubsection{Cost of service}

Each vehicle type has different costs associated with its use, due to fuel consumption, required staff, operation licenses requirements, maintenance and insurance. The total cost depends on the total time of use and the distance traveled. Hence, we can divide the cost in three different items: a fixed usage cost, a staff cost depending on the required time for the emergency operation, and a variable cost per kilometer. For each sanitary vehicle, we define the cost of service in Table 2, obtaining the total cost as the sum of the values for each 
Table 1: Average speeds of sanitary vehicles

\begin{tabular}{|l|c|c|c|}
\hline \hline \multirow{2}{*}{ Vehicle type } & \multicolumn{3}{|c|}{ Area type } \\
\cline { 2 - 4 } & Urban & Interurban & Rough terrain \\
\hline \hline Non-assistance Ambulance & $50 \mathrm{~km} / \mathrm{h}$ & $100 \mathrm{~km} / \mathrm{h}$ & $40 \mathrm{~km} / \mathrm{h}$ \\
\hline BLS Ambulance & $50 \mathrm{~km} / \mathrm{h}$ & $100 \mathrm{~km} / \mathrm{h}$ & $40 \mathrm{~km} / \mathrm{h}$ \\
\hline ALS Ambulance & $50 \mathrm{~km} / \mathrm{h}$ & $100 \mathrm{~km} / \mathrm{h}$ & $40 \mathrm{~km} / \mathrm{h}$ \\
\hline Fast Intervention Vehicle (FIV) & $60 \mathrm{~km} / \mathrm{h}$ & $120 \mathrm{~km} / \mathrm{h}$ & $70 \mathrm{~km} / \mathrm{h}$ \\
\hline Helicopter (HEH) & $250 \mathrm{~km} / \mathrm{h}$ & $250 \mathrm{~km} / \mathrm{h}$ & $250 \mathrm{~km} / \mathrm{h}$ \\
\hline \hline
\end{tabular}

Table 2: Cost of use of sanitary vehicles

\begin{tabular}{|l|c|c|c|}
\hline \hline \multirow{2}{*}{ Vehicle type } & \multicolumn{3}{|c|}{ Cost } \\
\cline { 2 - 4 } & Fixed & Medical Staff & Distance Traveled \\
\hline \hline Non-assistance Ambulance & $29 €$ & $36 € /$ hour & $0.68 € / \mathrm{km}$ \\
\hline BLS Ambulance & $29 €$ & $72 € /$ hour & $0.68 € / \mathrm{km}$ \\
\hline ALS Ambulance & $156 €$ & $81 € /$ hour & $1.56 € / \mathrm{km}$ \\
\hline Fast Intervention Vehicle (FIV) & $156 €$ & $45 € /$ hour & $1.56 € / \mathrm{km}$ \\
\hline Helicopter (HEH) & $468 €$ & $81 € /$ hour & $4.68 € / \mathrm{km}$ \\
\hline \hline
\end{tabular}

column. The data shown in Table 2 was obtained from an existing sanitary transport company in Spain (Amcoex, Inc., 2012).

\subsection{Sanitary vehicles allocation policy}

To correctly decide the sanitary resources required to assist a traffic accident, it is convenient to do an a priori classification of the accident in order to optimize the resource usage. In our model, accidents are classified and handled according to the percentage of injured passengers, including their corresponding severity (Fogue et al., 2012b). 
In general, there is no standardized method to allocate sanitary vehicles to accidents. Traditionally, resources are over-allocated, in anticipation of a particularly severe accident. However, some guidelines are provided in Medynet (2012), where it is always recommended that the rescue operation includes a nurse and/or doctor, and at least one of the ambulances should be an ALS. Thus, the medical staff will have the corresponding medical equipment to adequately assist serious injuries. However, for minor injuries, a BLS ambulance is enough to provide the required first aid. Therefore, each accident should be individually studied to define the required equipment and personnel depending on the severity of the accident. This issue will be included into our system by distinguishing three levels for accident classification:

- Minor accidents: Situations where there has been a minor collision and there is no risk of death for the occupants. In this type of accidents, there might be slight injuries among the passengers, but most occupants are considered unharmed. Since it is highly unlikely that this type of collisions need medical supplies beyond first aid, at least a BLS ambulance should be sent containing enough material for 3 passengers estimated to have minor injuries. The rest of the ambulances to be sent can be of the non-assistance type, allowing to evacuate the passengers to a hospital for further exploration. Many of these injuries will not even require transportation, being assisted in the accident site, so the allocated ambulances should be enough to transport $50 \%$ of the injured. We obtained this percentage by studying the accidents in the General Estimates System (GES) database maintained by the National Highway Traffic Safety Administration (NHTSA) (2012), showing that accidents with these characteristics usually present about $30-50 \%$ of people involved with possible injuries greater than the initially estimated. However, the percentage is mainly orientative and it could be adapted to better represent accidents happening in a specific area if we had enough information collected using our system. 
- Intermediate accidents: These accidents are not the most severe ones, although they may be potentially lethal for some of the passengers. Often, most passengers suffer from minor injuries, but there is at least one of them with a high probability of severe injuries. The rescue operation should include an ALS unit with material for every 3 injured passengers, as well as enough additional ALS vehicles to correctly evacuate the severely injured occupants individually. Due to the higher probability of dangerous injuries, it would be convenient to send enough BLS vehicles to transfer at least $75 \%$ of the rest of injured passengers. Again, the selection of this percentage is based on data from the GES database.

- Severe accidents: They represent the highest severity for a traffic accident. The collision was important enough to produce very severe injuries to the passengers. Hence, most of the occupants in the vehicles are estimated as severely injured. The rescue operation requires sending ALS vehicles for every passenger with potentially lethal injuries. Additional ALS units with support material should also be sent for every 3 people with minor injuries. To avoid unexpected complications on the affected passengers, all of them should be transfered to the nearest hospital by using BLS vehicles.

The selected resources for each accident will also depend on the conditions of the accident itself. Hence, for minor or intermediate accidents, the use of rescue helicopters should only be acceptable if they occur in areas located very far away from health centers. If the accident happens in a poor access area, it could be convenient to send a FIV vehicle in charge of the stabilization of the affected people before the arrival of other emergency vehicles.

Another possibility consists of sending vehicles with less assistance category than needed, due to the proximity to the crash site. This would be beneficial if it is possible to correctly evacuate the injured passengers to a health center faster than the required resources would need to reach the accident. 


\subsection{Objectives of the resource allocation}

The greatest difficulty of the resource allocation problem resides in the different conflicting objectives when obtaining an optimal set. We would want to select the nearest resources from the accident area, but this is not always possible due to the cost of some of the resources: sending a helicopter to an accident without serious injuries would not be affordable, even though it is the fastest of all the available vehicles. Hence, the optimal solution must offer a balance between different objectives, often contradictory. We defined the following objectives for traffic accident assistance:

- Assistance quality: The first and most obvious of the objectives to be met is based on the assistance quality received by those injured in the accident. This parameter is measured in terms of the time it takes for medical teams to access the crash site, as well as the type of injuries they are able to attend. Thus, if a seriously injured passenger requires advanced life support to maximize his survival chances, and the system selects a basic life support equipment instead, this should be reflected in the assistance quality with a penalty.

- Cost: The second parameter to consider is the cost of the rescue operation. As we showed in the classification of resources, the cost for each resouce can be subdivided into several elements: licenses, insurance, travel, staff, etc. The value used to represent the cost will come from the sum of the individual costs of all vehicles, with their corresponding medical staff, dispatched to the scene of the accident.

- Reduced resource overuse: To avoid excessive wearing out of the equipment and vehicles, and fatigue in medical personnel involved in rescue missions, the equipment and human resources of the different suppliers should be used in the most equitable manner possible. This parameter has lower priority, since the care of the equipment should never overcome the health of the passengers, but there may be situations where different solu- 
tions provide similar assistance quality, and then this parameter may be used to choose between one or the other.

- Balanced resource deployment: The last factor to be considered avoids having any of the existing health centers deprived from all its resources at a given time, thereby preventing situations where a serious accident could occur in the vicinity.

It is easy to observe that these objectives are contradictory, since increasing the quality of the assistance to the injured is often only possible by increasing the cost of the emergency operative. The most adequate solution to the problem should take into account all these factors to reach an acceptable balance, that will also depend on the scenario and the economical and social environment.

\section{Multi-objective Optimization: Search through Genetic Algorithms}

The problem of resource allocation to traffic accidents is considered an optimization problem, since we know the model representing the system, and the goals to achieve. So, the objective is to find the input that achieves the given goals, i.e., the set of selected resources to be sent to the accident site. However, the goal is not simple, since we have four different objective functions: assistance quality, cost, reduced resource overuse, and balanced resource deployment. We propose to address this issue using an optimization problem approach following a multi-objective optimization based on genetic algorithms.

The rest of the Section is organized as follows: Section 3.1 presents the different existing approaches to multi-objective optimization. Section 3.2 introduces the evolutionary algorithms with their main features. Finally, Section 3.3 shows why evolutionary algorithms are appropriate for multi-objective optimization problems.

\subsection{Multi-objective Optimization}

The problem we face has a special feature compared to standard optimization approaches, where there is only one objective function to optimize and selecting 
the best solution is the common approach. Multi-objective problems are those having several objectives which are usually mutually exclusive, so that all of them can not be optimized at the same time. Those kind of problems are very common in engineering, where in most cases the performance or reliability is maximized with minimum cost.

Several approaches can be followed to address multi-objective optimization problems. The straightforward method consists on combining the individual objective functions into a single composite function, using methods such as utility theory or weighted averages. This approach has the advantage of being simple, not requiring changes in the optimization algorithm used with a single objective function. However, the selected weights or utility functions must be carefully tuned to characterize the preferences of the entity responsible for making decisions. In addition, very small changes in the weights may lead to very different solutions.

However, during a decision-making process, it may be preferable to have a good set of potential solutions on which to decide which better address each particular situation, rather than having just a single solution as obtained with the methods presented so far. This approach relies on generating a Pareto set of optimal solutions, also called Pareto front or Pareto frontier (Teich, 2001).

The concept of Pareto efficiency was initially defined in the area of economy. According to Pareto, a situation $Y$ is superior or preferable to a situation $X$ when the change from $X$ to $Y$ is an improvement for all the members of the society; or an improvement for some, but no one is adversely affected (Pareto, 1909). This concept can be also applied to multi-objective optimization problems. Therefore, a solution $S 1$ is Pareto-optimal when no other solution $S 2$ improves an objective without worsening at least one of the other objectives. This is defined on the concepts of Pareto dominance and Pareto optimality:

- Pareto dominance: Given a vector $\mathbf{u}=\left(u_{1}, \cdots, u_{k}\right)$, it dominates another vector $\mathbf{v}=\left(v_{1}, \cdots, v_{k}\right)$ if:

$$
\forall i \in\{1, \cdots, k\}, u_{i} \leq v_{i} \quad \wedge \exists i_{0} \in\{1, \cdots, k\} \mid u_{i_{0}}<v_{i_{0}}
$$


- Pareto optimality: A solution $\mathrm{x}^{*}$ is Pareto-optimal if there is no different vector $\mathbf{x}$ so that $\mathbf{v}=f(\mathbf{x})=\left(v_{1}, \ldots ., v_{k}\right)$ dominates $\mathbf{u}=f\left(\mathbf{x}^{*}\right)=$ $\left(u_{1}, \ldots, u_{k}\right)$.

In general, the solution to a problem of multi-objective optimization is not unique: the solution will consist of the set of all non-dominated vectors, which are known as the non-dominated set, Pareto front or Pareto frontier. The Pareto frontier may have different sizes (even infinite), and the Pareto set size usually grows while increasing the number of objective functions (as a result of increased dimensionality of the problem). As a result, obtaining the full set of optimal solutions is not feasible for many problems. Thus, for practical purposes, the aim is to investigate a rough set of solutions that represent the best possible Pareto optimal set, leading to several conflicting objectives (Zitzler et al., 2000):

- The approximate Pareto frontier should be as close to the true Pareto set as possible. Ideally, this approximate boundary should be a subset of the actual border. This requires intensifying the search in a particular region of the Pareto frontier.

- Solutions in the rough set should be evenly distributed along Pareto frontier, to provide decision-makers a clear picture of the sacrifices to be made to achieve the different solutions.

- The approximate set should capture the full spectrum of the Pareto frontier, which requires investigating the solutions at the ends of the space of objective functions, extending the set of Pareto.

In the existing literature, we can find different analytical and numeric algorithms to approximate the Pareto front, such as Normal Boundary Intersection (NBI) (Indraneel and Dennis, 1998), Normal Constraint (NC) (Messac et al., 2003; Messac and Mattson, 2004), and Directed Search Domain (DSD) (Erfani and Utyuzhnikov, 2010). However, these methods present several shortcomings due to the significant number of redundant solutions that they can generate 
(Utyuzhnikov et al., 2009), and the problems that arise when the Pareto frontier is not continuous (Shukla and Deb, 2007). Nowadays, most of the existing related work about multi-objective optimization is based on the use of evolutionary algorithms, which could overcome those important drawbacks.

\subsection{Evolutionary algorithms}

Evolutionary algorithms have the ability to simultaneously search different regions of the solution space, including those with non-convex spaces, discontinuous, or multi-modal. Although there are different variants of evolutionary algorithms, the idea behind them all is the same: given a population of individuals, the environmental pressure produces a natural selection process that causes an increase in the adaptation level of the individuals to the environment. For each generation, some of the best candidates are chosen to create a new generation by applying the recombination and mutation operators, producing new individuals that will compete again in the environment. The general scheme of an evolutionary algorithm (Eiben and Smith, 2003) is shown in Algorithm 1.

The recombination or crossover operator is usually the most important factor in this type of algorithms. It is based on combining two or more genotypes, i.e., gene sequences, called parents to form new genotypes, the offspring. Parents are selected from existing individuals based on their level of adaptation or fitness, so you can expect that the offspring inherits the good genes that make their parents the fittest. Applying iteratively this operator, it is more likely that the best chromosome genes appear more frequently in the population, ultimately leading to a convergence towards the best solutions.

The mutation operator is responsible for introducing random changes in the characteristics of the chromosomes, and it is usually applied at the gene level. As a rule, the mutation rate (probability of change in a gene) is usually very small and dependent on the length of the genotype, so that the new genotypes produced after the mutation will not be very different from the originals. The role of the mutation in the evolutionary algorithms is also very relevant, since the recombination operator makes the individuals converge rapidly toward the 


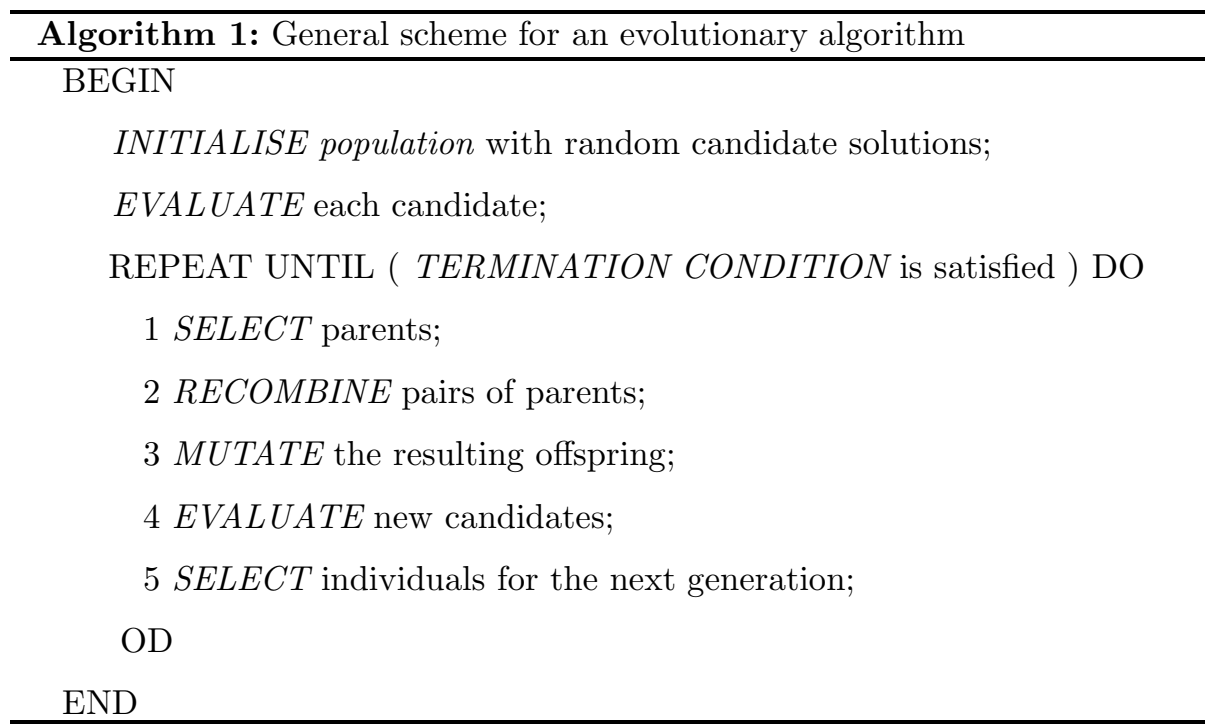

best solutions found so far. However, there may be a region of the state space not previously explored, and so the mutation is used to reintroduce genetic diversity in the population, thereby avoiding local optima.

\subsection{Multi-objective Optimization based on Evolutionary Algorithms}

Through an appropriate use of the recombination and mutation operators of evolutionary algorithms, it is possible to obtain good solutions with respect to different objectives, obtaining new non-dominated solutions in the Pareto frontier. In addition, most of the developed evolutionary algorithms do not require the objective functions to be prioritized over each other, to be scaled, or to use weights to find weighted aggregate functions. For that reason, most optimization algorithms are multi-objective meta-heuristics based on an underlying evolutionary algorithm (Jones et al., 2002).

The classical (and straightforward) approach to the problem of multi-objective optimization was based on assigning a weight $\left(w_{i}\right)$ for each normalized objective function $\left(f_{i}^{\prime}\right)$, so the problem is reduced to a single objective formed as the weighted sum of these values. This method is known as a priori, since the user has to supply the values for the weights. A unique solution is obtained for 
each weight vector. However, as discussed above, the major drawback of this approach is that small variations in the weight vector can lead to completely different solutions.

More sophisticated versions of these algorithms approximate the Pareto frontier using different approaches. The first multi-objective genetic algorithm that appeared to approximate the Pareto frontier was the Vector Evaluated Genetic Algorithm (VEGA) (Schaffer, 1985). VEGA divides the population into subpopulations and assigns a different objective function to each of them. This approach could be easily implemented; however, the solutions tend to converge towards the extremes of the objective functions, achieving good results for one function but behaving very poorly for the rest.

The posterior Multi-Objective Genetic Algorithm (MOGA) (Fonseca and Fleming, 1993) uses the fitness sharing concept, so that the search towards unexplored sections of the Pareto frontier is favored by penalizing the fitness value of the solutions in densely populated areas. The fitness value is obtained by assigning ranks to the different solutions, depending on whether they are in the Pareto front, and their distance to it. MOGA is an extension of a standard genetic algorithm, but it has slow convergence and requires additional parameters specification, which is not always easy and may require several tests to find them.

The Pareto Envelope-based Selection Algorithm (PESA) (Corne et al., 2000) divides each dimension of the objective space into cells, where the cell size is defined by the user. The number of solutions in each cell is defined as the cell density, and this density information is used to achieve diversity in the individuals. PESA is easy to implement and efficient, but its performance depends on the size of the cells in each dimension, requiring prior knowledge of the objective space to decide how to divide it efficiently. The PESA-II extension (Corne et al., 2001) selects cells instead of individual solutions, but the cell size problem persists.

The Nondominated Sorting Genetic Algorithm (NSGA) (Srinivas and Deb, 1994) is a fitness-sharing algorithm characterized by its fast convergence towards 
the Pareto front. The population is classified as non-domination fronts, and each one receives a value of fitness that penalizes the nearest solutions. Similarly to other methods based on fitness sharing, it is difficult to adjust the parameters. To overcome this problem, a new version called NSGA-II (Deb et al., 2002) was proposed, which employs a crowding distance for a uniform distribution of the solutions in every front. This algorithm is very efficient and does not require additional parameters in the search, although the concept of crowding distance can be only applied to the objective function space, not to the solution space.

The different algorithms proposed were designed to guide the evolutionary process in order to obtain the optimal solution to a specific problem, being the NSGA-II algorithm, in theory, the most efficient variant in the literature. However, we still need to define the rest of the parameters needed to achieve proper results, such as the representation of individuals, and the crossover and mutation operators.

\subsection{Hybridization with other techniques: Memetic Algorithms.}

In practice, evolutionary algorithms are frequently applied to a problem in which a considerable amount of experience and knowledge is available. This information can then be used as specialized operators to produce performance benefits. In these cases, it is usual that the combination of evolutionary algorithm and a heuristic method performs better than either of the two original algorithms separately.

This type of hybrid algorithms, combining evolution with heuristics, are often based on the idea of "memes" (Dawkins, 1976), which can be seen as units of cultural transmission, in the same way that genes are units of biological transmission. These memes are selected according to their utility, and transmitted through interpersonal communication. The idea of using information not encoded in the genes, increasing the evolutionary search process with different local search processes, makes these hybrid algorithms to be often known as Memetic Algorithms (MA) (Moscato, 1989). 
Hybridization of genetic algorithms with local search algorithms are frequently applied in single-objective approaches. Generally, a local search algorithm proceeds as follows: (i) start with an initial solution $x$, (ii) generate a set of neighboring solutions around the solution $x$ using a simple rule of variation, and (iii) if the best solution on the set of neighbors is better than $x$, replace $x$ with this solution and return to the second step, otherwise stop the search.

Hybridization in the multi-objective algorithms has not been extensively studied so far. There have been only few attempts in problems with two objective functions (Paquete and Stutzle, 2003) or applying local search only to the final solutions to ensure their dominance (Deb and Goel, 2001). A more comprehensive attempt to generate a multi-objective memetic algorithm was developed in Knowles and Corne (2000), which presents the algorithm M-PAES (memetic version of PAES), employing the concept of dominance to evaluate local solutions. When a neighboring solution is created, it is only compared with the set of nondominated solutions. The local search is finished after a number of local movements without any improvement.

\section{Genetic algorithm for sanitary resource allocation}

We propose a novel method to improve the resource allocation process in a traffic accident: Genetic Algorithm for Traffic Accidents Resource Allocation (GATARA). Our model will provide a set of resources (final solution) to be sent to the accident area, based on the necessary resource types defined by the allocation policy presented in Section 2.

To accomplish that, our model makes use of the Genetic Algorithm (GA) subtype among the general Evolutionary Algorithms. In the problem of sanitary resource allocation for traffic accidents, we must consider that the final solution will consist of a set of selected resources, and therefore the search process should determine, for each available resource, if it is sent or not. We are facing a problem where we must be decided for a number of elements, which can be represented in a vector, whether they are selected or not. This situation 
could be expressed as selected $=$ true or selected $=$ false. In other words, each possible solution can be represented by a vector of Boolean values, and each element is associated with a resource. The Genetic Algorithms (GA) represent the candidate solutions as strings over a defined alphabet, making them the most appropriate type of algorithm.

Our algorithm is based on the execution cycle performed by the Nondominated Sorting Genetic Algorithm, Fast version (NSGA-II) (Deb et al., 2002), which allows efficiently searching the solution space to generate uniform Pareto front approximations. However, we will include a hybridization of this algorithm to improve its convergence speed towards the optima. The results of our proposal will be compared with other genetic algorithms that could be adopted, making use of the simple a priori approach, or approximating the Pareto front: Vector Evaluated Genetic Algorithm (VEGA) (Schaffer, 1985), and Multi-Objective Genetic Algorithm (MOGA) (Fonseca and Fleming, 1993).

\subsection{Parameter Definition for the Genetic Algorithm}

Our genetic algorithm require a series of components that need to be defined to determine their functioning, which are dependent on the specific problem to solve. In our case, we must adapt the general parameters of genetic algorithms to the sanitary resource allocation problem.

\section{- Representation of individuals:}

The first step in defining the algorithm is to determine how to represent realworld situations in our genetic algorithm. The representation of the individuals is the link between genotypes, i.e., chromosomes containing genetic information for each of the individuals, and the phenotypes, the different solutions to the original problem.

The phenotype for the search of medical resources consists of a set of vehicles to be sent to the crash site for treatment and stabilization of the injured before being evacuated to hospital to complete their assistance. Each individual, i.e., possible solution, is represented in the population by using a vector of bits where 
Available resources:

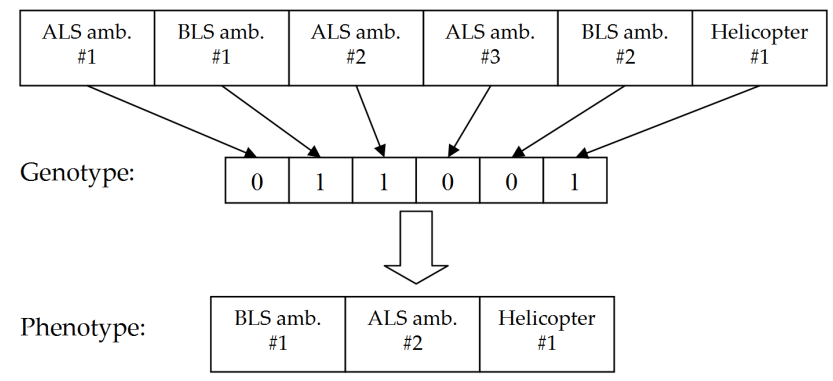

Figure 2: Representation of individuals in the genetic algorithm for resource allocation.

each position is associated with an available resource. A value of 1 indicates that the resource associated to that position is selected; otherwise, the value in the vector is 0 . Figure 2 shows an example with six available resources.

\section{- Evaluation functions:}

When determining the most appropriate sanitary resources for a traffic accident, we must take into account that different providers may have different priorities, being the closest resources not always the most appropriate ones. There are many factors that may be important in the decision of the final set. The objective functions used are designed to transform the problem in a minimization one, and hence greater values are considered as penalties and they should be reduced. The four evaluation functions defined are:

1. Assistance quality penalty $\left(f_{1}\right)$ : The assistance quality is measured as the time required for the medical teams to access the crash site, and the type of injuries they are able to handle. Thus, if a serious injury requires advanced life support to maximize their survival chances, and if our algorithm selects a basic life support equipment, the assistance quality should be penalized. Since the rescue time is the most critical factor in a traffic accident, the penalty in the assistance quality due to the time past since the notification of the accident will be quadratic. In addition, we differentiate between two types of resources: transport vehicles, needed to 
transfer the injured passengers to a hospital in a safe way; and support vehicles, that provide additional equipment and medical staff. The arrival time of the sanitary vehicles is computed using a map representing the streets and roads around the crash site. Road vehicles are supposed to use the shortest path from their locations to the accident area, whereas helicopters use a straight line for their route. Algorithms 2 and 3 show our proposed scheme, which is used to assign assistance quality penalties for each type of resource. These algorithms allow obtaining the final value for the assistance quality function, defined as:

$f_{1}(R)=\sum_{i=1}^{m} w_{i} \cdot$ assistance_quality $\left(R, p_{i}\right)+\sum_{j=1}^{n} w_{j} \cdot \operatorname{support} \_q u a l i t y\left(R, a_{j}\right)$

Where $R$ is the set of selected resources, $m$ is the number of passengers $\left(p_{i}\right), n$ is the number of required support resources (with assistance category $a_{j}$ ). The weights $w$ depend on the category requested, being defined as 1 for non-assistance vehicles, 2 for BLS resources, and 4 for ALS vehicles to reflect the higher importance of ALS equipment for severe injuries.

2. Cost $\left(f_{2}\right)$ : The total cost of the rescue operative, representing the overall cost of all the individual resources selected to be deployed at the crash site. The values to determine the cost of each resource are obtained from Table 2, and it is calculated using Equation 3, where $n$ is the number of selected resources.

$$
f_{2}(R)=\sum_{i=1}^{n} \operatorname{cost}\left(r_{i}\right)
$$

3. Resource overuse penalty $\left(f_{3}\right)$ : In an ideal situation, the resources from the different suppliers would be used in an equitable manner to avoid an overusing on the equipment and vehicles, as well as reducing fatigue in the medical personnel. The calculation of this value is done by determining the time elapsed since the last time when the resource was used. The smaller this value, the greater the penalty provided by the resource when used again. Equation 4 shows how this function is calculated for $n$ selected resources and the time being indicated in hours. 


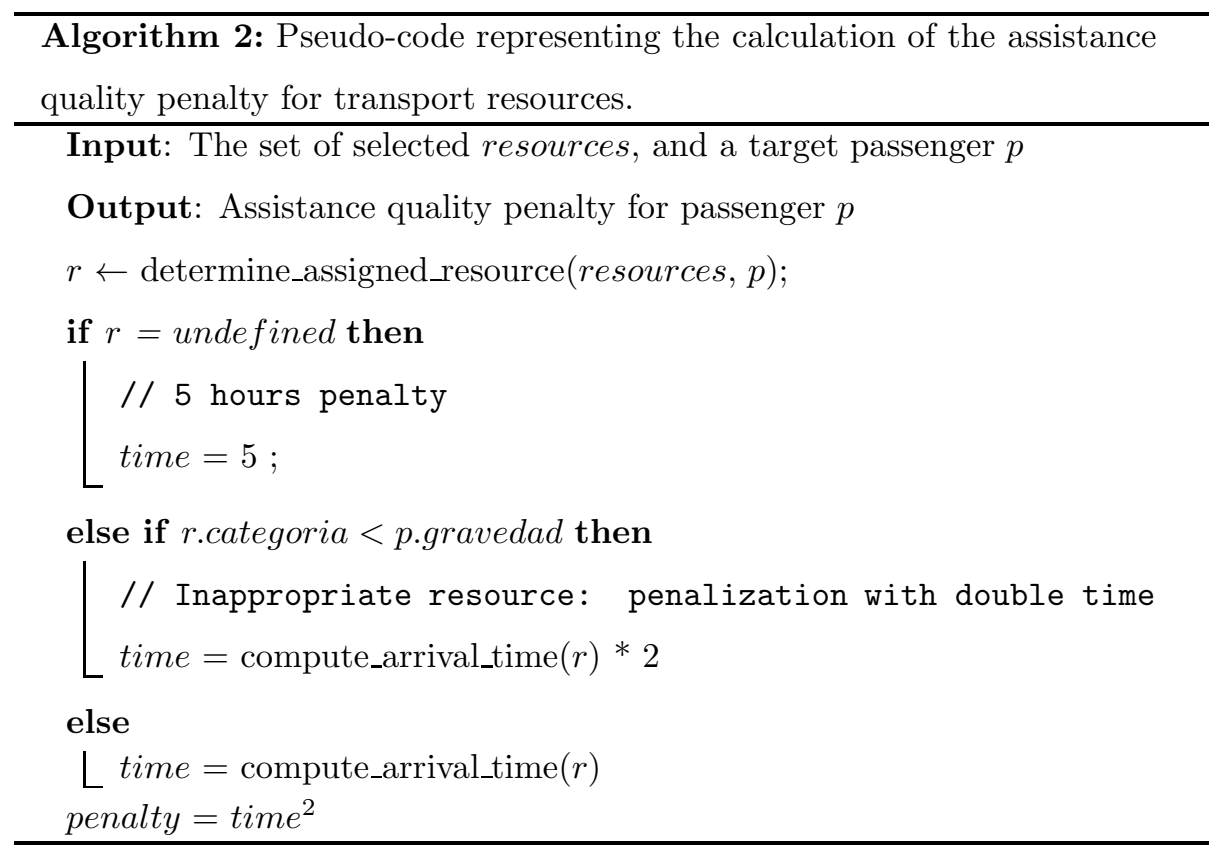

$$
f_{3}(R)=\sum_{i=1}^{n} \frac{2}{\text { current_time }-r_{i} \text { last_use_time }+2}
$$

4. Emergency threshold penalty $\left(f_{4}\right)$ : This function tries to avoid health care centers to be deprived of all their resources at any given time, so maintaining their clinical operatives in case of nearby accidents. Each health care center has thresholds defined for BLS and ALS resources, and a penalty is added if the number of resources available is lower than the corresponding threshold. The final value is the sum of the penalties for each of the $k$ health centers:

$$
\begin{gathered}
\text { thr_penalty }(h c)=0.4 \cdot \frac{b l s_{-} t h r-b l s_{\_} \text {units }}{b l s_{-} t h r}+0.6 \cdot \frac{\text { als_thr }- \text { als_units }}{\text { als_thr }} \\
f_{4}(R)=\sum_{i=1}^{k} t h r_{-} \text {penalty }\left(h c_{i}\right)
\end{gathered}
$$

\section{- Population:}




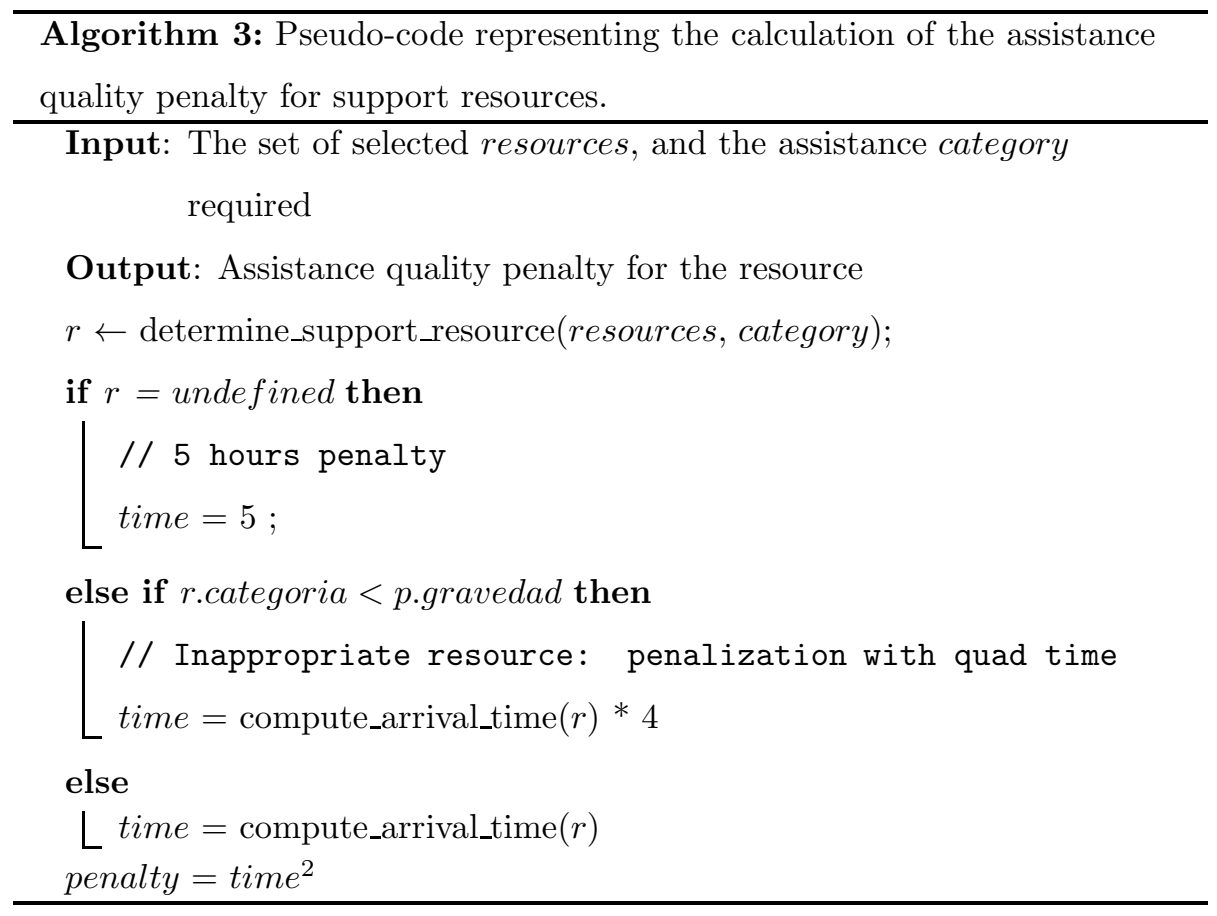

The population contains the possible candidate solutions to be evaluated at a particular time (generation). We will use populations with a fixed number of individuals, which have been commonly used. Although there are algorithms with a variable number of individuals in the population (Affenzeller et al., 2007), the benefits are purely spatial, since it allows storing fewer individuals in memory. However, the convergence speed of the algorithm does not change significantly.

In general, the population size influences the speed of the problem solving algorithm. Reducing the population size increases the speed optimization to some extent, from which a premature convergence occurs reducing the speed (Koljonen and Alander, 2006). In addition, the reliability of the optimization, i.e., the ability to find the optimal value, normally increases monotonically with the size of the population. We selected 10 individuals for populations based on the a priori approach, and 30 individuals when the objective is to find the Pareto front, since more solutions should give a more detailed idea of the shape of the frontier. 


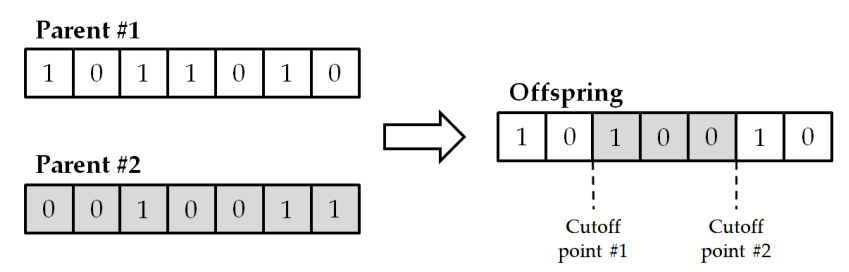

Figure 3: Example of crossover operator using two cutoff points.

\section{- Parent selection:}

The main purpose of the parent selection phase is to determine which individuals are best suited to have children and pass their genes to the next generation. Most algorithms (such as VEGA, MOGA and a priori approaches) do not define a specific mechanism for parent selection. However, NSGA-II is specifically designed to use a binary deterministic tournament mechanism $(k=$ 2) (Bäck, 1996). In the comparisons of the different algorithms, all of them will be configured to make use of this mechanism.

\section{- Crossover operator:}

Crossover or recombination is a binary operator that joins the information from the genotypes of two parents in one or more offspring genotypes. There are several types of recombinations in genetic algorithms. The classical operator has been the 1-point crossover. Given two parent genotypes, a cutoff point is chosen and the offspring genotype genes take values from the first parent before the cut, and from the second parent after the cut. In our case, we will employ an extension of this scheme using two cutoff points, in order to increase the variability on the genotype of the offspring, as shown in Figure 3.

\section{- Mutation operator:}

Mutation in genetic algorithms has been traditionally defined as the probability of change for a single gene. This probability is usually low to avoid excessive changes in the offspring that could move the individual away from the 
area that it is currently exploring, but this mechanism should exist to successfully avoid local optima. In our proposal we will use a probability of 0.02 , i.e., one change for every 50 genes on average.

\section{- Survival selection:}

The selection of survivors, or environmental selection, is aimed at selecting individuals depending on their quality to create the next generation. It is usual to use a standard generational replacement, where the new offspring completely replaces the individuals from last generation. However, NSGA-II and GATARA are elitist schemes, thus the best individuals from the last generation are included in the next generation to keep track of the best solutions found so far.

\subsection{Constraint handling}

In optimization problems where the variable values are not subject to any restriction, i.e., those in which the variables can take any value in the domain, the concept of Pareto dominance presented so far is sufficient to compare two solutions. However, most engineering problems have restrictions that may cause some of the obtained solutions to be unfeasible.

If we analyze our problem of resource allocation, all possibilities are feasible in terms of sets of resources to be sent to an accident area. However, some of them are clearly more suitable than others. The simplest example is the solution that sends no resources to the crash site. It is easy to see that this solution will never be dominated by any other, since the objective functions based on cost, overuse penalty, and emergency threshold violation would present the minimum value, although the assistance quality will be null. Therefore, we must prevent solutions excessively neglecting the assistance of the injured.

An interesting approach (Deb et al., 2002) makes use of the constraintdominance concept, where a solution $i$ dominates another solution $j$ if any of these conditions is true:

1. Solution $i$ is feasible, while solution $j$ is not. 
2. Both solutions are unfeasible, but solution $i$ presents less violation to the feasibility condition.

3. Both solutions are feasible, but solution $i$ dominates over $j$.

In our algorithm for sanitary resource allocation, a solution is considered feasible if it provides at least the amount of resources required by the accident using the proposed allocation policy, even when their assistance category is not optimal. That is, the number of vehicles sent should allow evacuating all the person according to the allocation policy, which also depends on the accident severity estimation. Thus, the solution of not sending any resources will be dominated by any other solution subject to these restrictions. Between two unfeasible solutions, the solution able to evacuate a greater number of occupants is preferred. Finally, from two feasible solutions, we would select the Paretodominating one.

\subsection{Hybridization of the NSGA-II Algorithm in GATARA}

The original NSGA-II algorithm could be improved by adding knowledge about the problem that could help to find better solutions in fewer generations. When a hybrid, or memetic, multi-objective algorithms is developed, we should take into account the solutions to which local search will be applied, and how to identify a solution in the vicinity as the new best solution when there are multiple local non-dominated solutions.

Generating neighboring solutions to the problem at hand can be simple using inversion of bits, or bit-flipping, since the new solution only differs from the original one in a single resource to be added or removed from the rescue team. Moreover, local search must be applied selectively to be computationally efficient. Hence, instead of checking all solutions of the offspring, GATARA only investigates those that are not dominated by any other solution, also know as rank 1 solutions, after all the solutions have been assigned to different domination fronts in NSGA-II. The local search process will finish when the first better solution is found in the vicinity, or when there are no more neighbors left to explore. 
Table 3: Parameters for the Resource Allocation Genetic Algorithm

\begin{tabular}{|l|l|}
\hline \hline Representation & Binary strings \\
\hline Recombination & 2-point crossover \\
\hline Recombination probability & $90 \%$ \\
\hline Mutation & Bit-flipping \\
\hline Mutation probability & $2 \%$ \\
\hline Parent selection & Tournament $k=2$ \\
\hline Survival selection & Generational \\
\hline Population size & 10 ( a priori $), 30$ (Pareto Front approach) \\
\hline Initialization & Random \\
\hline Termination condition & 200 generations ( $a$ priori $), 300$ generations \\
& $($ Pareto Front approach $)$ \\
\hline Number of executions & 20 \\
\hline \hline
\end{tabular}

\section{Algorithm evaluation}

The proposed model will now be evaluated using a series of pre-generated scenarios representing accidents occurring in different situations. The data for the accidents is taken from the GES database to represent realistic situations.

\subsection{Definition of the evaluation problem}

To evaluate the chosen algorithms, we generated two situations in which, for the same accident, the number of providers is different. The specific accident is a multiple collision set in an interurban road which took place in the minute 3,000. The potential resources for the accident are searched in the $10,000 \mathrm{~km}^{2}$ surrounding area. That is, we consider a $100 \mathrm{~km} \times 100 \mathrm{~km}$ square area in which the accident is located in the central coordinates $(50,50)$. The collision involved the following vehicles and passengers:

- A tourism with severe damage estimation. Three passengers were in the 


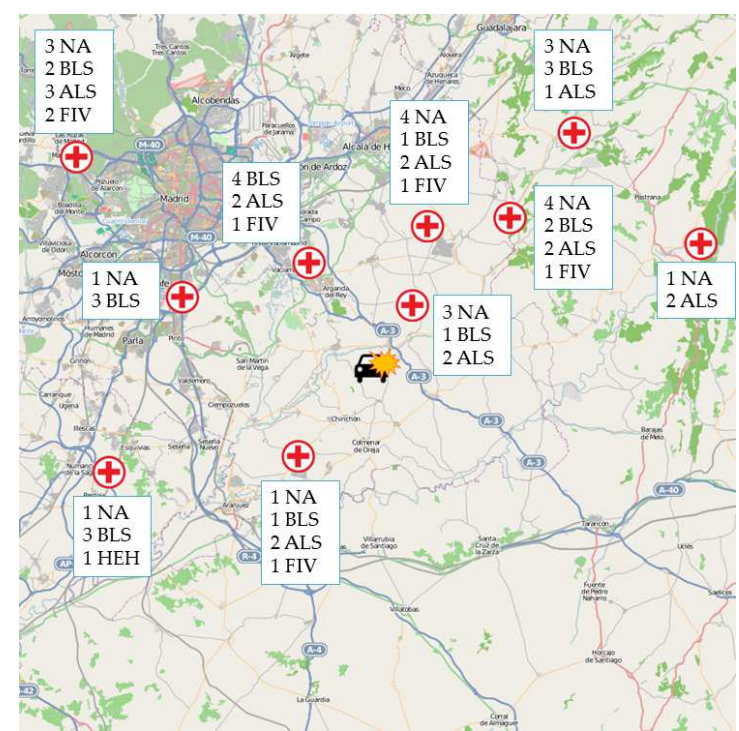

(a)

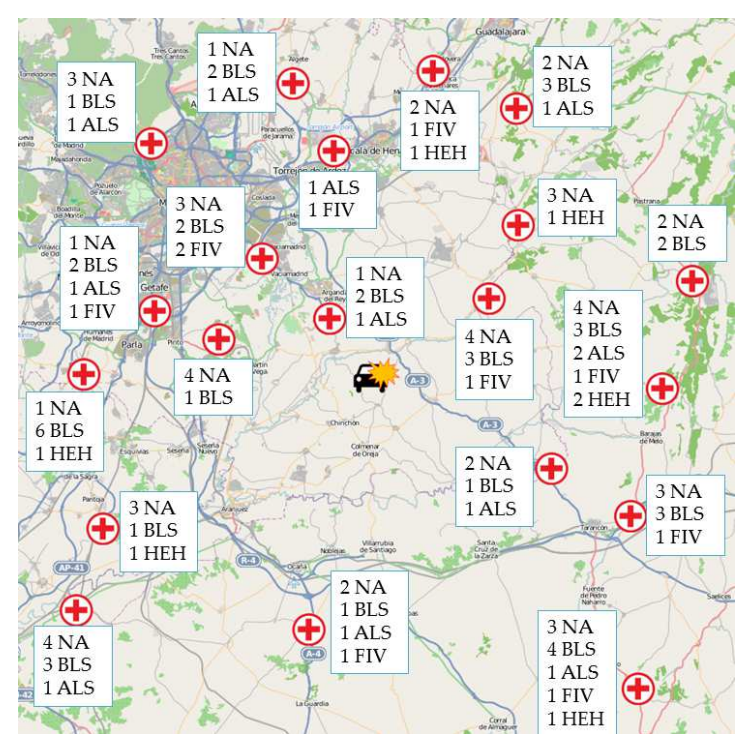

(b)

Figure 4: Example scenarios for a traffic accident resource allocation in a $100 \mathrm{~km} \times 100 \mathrm{~km}$ area with (a) 10 suppliers, and (b) 20 suppliers. 
vehicle at the time of the accident, where two of them are estimated to present severe injuries, and the third one just minor injuries.

- A truck with minor damage, having the driver as the only passenger, with minor injuries estimation as well.

- A small vehicle with severe damage, occupied by 3 passengers with severe injuries estimation.

According to our resource allocation model, the incident would be classified as severe, and the estimated resources for assistance would consist of:

- 5 Advanced Life Support vehicles for the possible transport of the seriously injured in the collision.

- 2 Basic Life Support vehicle for the possible transport of minor injured passengers.

- 1 Advanced Life Support vehicle to support BLS vehicles (for example, to provide additional material).

In our case, our scenarios will include several sanitary resource suppliers, such as rescue centers and hospitals. As shown in Figure 4, we generated two random scenarios with potential suppliers around the crash site: the first one includes 10 suppliers, and the second one involves 20 suppliers. The available resources for each of them were also generated randomly. The map was obtained from the area around Madrid (Spain), using OpenStreetMap (OpenStreetMap, 2012). The parameters of the executions of the selected genetic algorithms are shown in Table 3.

\subsection{Drawbacks of the simple a priori approach}

The straightforward method for adapting a genetic algorithm to be multiobjective is to generate a new function resulting from the combination of the multiple chosen objective functions. We may perform a weighted mean using the normalized values from the four functions, with a set of weights varying 
Table 4: Weight sets used in the a priori approach

\begin{tabular}{|l|c||c|c|c|c|c|}
\hline \hline \multirow{2}{*}{ Objective Function } & \multirow{2}{*}{ Weight } & \multicolumn{5}{|c|}{ Weight set \# } \\
\cline { 4 - 8 } & & $\mathbf{1}$ & $\mathbf{2}$ & $\mathbf{3}$ & $\mathbf{4}$ & $\mathbf{5}$ \\
\hline \hline Assistance Quality Penalty & $w_{1}$ & 0.7 & 0.5 & 0.5 & 0.4 & 0.3 \\
\hline Cost & $w_{2}$ & 0.15 & 0.2 & 0.2 & 0.4 & 0.5 \\
\hline Resource Overuse Penalty & $w_{3}$ & 0.075 & 0.05 & 0.25 & 0.1 & 0.1 \\
\hline Emergency Threshold Penalty & $w_{4}$ & 0.075 & 0.25 & 0.05 & 0.1 & 0.1 \\
\hline \hline
\end{tabular}

depending on the relative importance of each function. Hence, the fitness value for each individual $i$ of the population at time $t$ will be obtained through the following formula:

$$
f(i, t)=\sum_{j=0}^{4} w_{j} \cdot f_{j}(i, t)
$$

where $w_{j}$ represents the weight assigned to the objective function $f_{j}$.

We performed several executions using the weight sets included in Table 4, which represent different allocation policies with more preference for one or more objective function. For example, the weight set 1 presents a clear preference towards assistance quality, while the weight sets 4 and 5 try to achieve a balance between assistance quality and cost, probably indicating a situation where the cost could be highly reduced. These weights must be manually included into the algorithm, meaning that the specific weight for each function is an approximation of the intentions of the entity in charge of the decision.

The optimal solutions found using the a priori approach after 20 executions of 200 generations are shown in Figure 5. The differences between the five weight sets presented are very noticeable. Even when two weight sets that are not too different, like sets 2 and 3, where only the two functions with less priority change their weight values, totally different solutions are obtained, making it very difficult to adjust them to represent a given allocation scheme. We can observe in Figure 5(a) how the values for the functions change noticeably for 


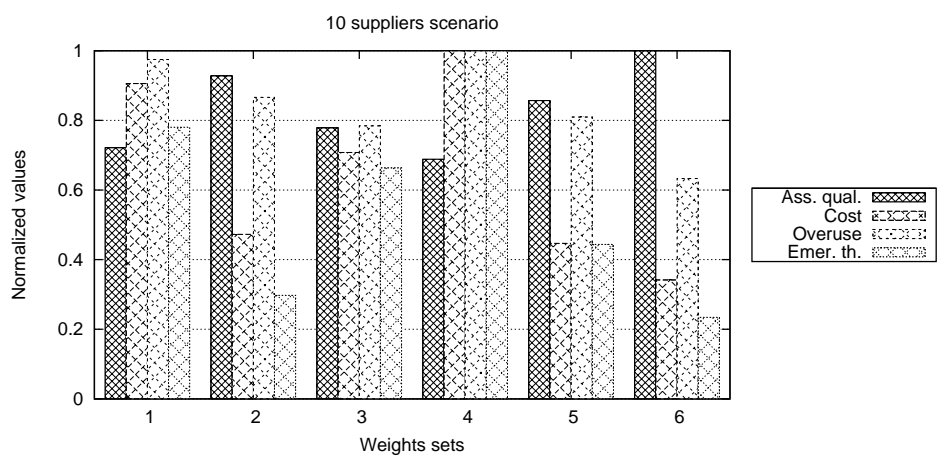

(a)

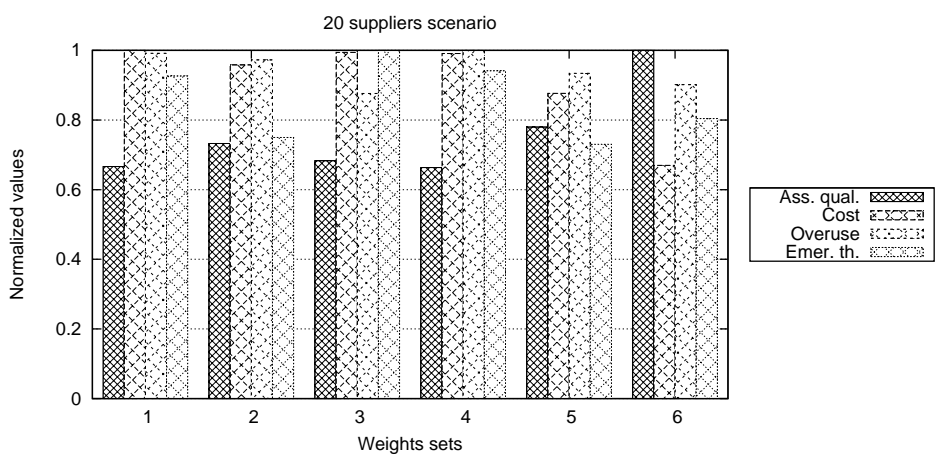

(b)

Figure 5: Different solutions obtained using different weight sets for the a priori approach in the (a) 10 suppliers scenario, and (b) 20 suppliers scenario. 
them, obtaining a totally different shape in the graph bar. A similar effect is appreciated when studying weight sets 4 and 5 , where the cost function is reduced by greatly increasing the assistance quality and the overuse penalty.

In addition, comparing Figures 5(a) and 5(b), it is shown that the differences between the solutions found is more important when fewer suppliers are taken into account. Therefore, having less options makes the solution vary significantly, which may be a problem in areas with few health centers available. This undesirable effect makes this simple scheme less useful when we need to define a precise allocation policy, and using the Pareto front approach becomes an option to overcome this drawback.

\subsection{Comparison between GATARA and other algorithms approximating the Pareto front}

Given the limitations observed in the a priori approach for multi-objective search, we conclude that it is more beneficial to present a set of non-dominated solutions representing the Pareto front, instead of just selecting a single solution with the a priori approach. Our proposed scheme GATARA is based on the NSGA-II algorithm, and we will compare it to the original version of this algorithm, as well as other existing multi-objective genetic algorithms: VEGA and MOGA. These algorithms will be evaluated using the scenarios previously presented, both with 10 suppliers and 20 suppliers.

Figure 6 shows the results obtained with the four algorithms in the 10 suppliers scenario. The data series indicate the mean value of the four objective functions while increasing the number of generations of the population. The three schemes obtain very similar results in terms of assistance quality and cost. It is noticeable how they treat the assistance quality, since VEGA and MOGA are approximately constant during all the execution, whereas NSGA-II and GATARA worsen their results during the first generations to explore the solutions space, and later they stabilize with results that are similar to the other two algorithms. There are more differences related to the overuse penalty function, with clear advantage for GATARA, and the emergency threshold penalty 


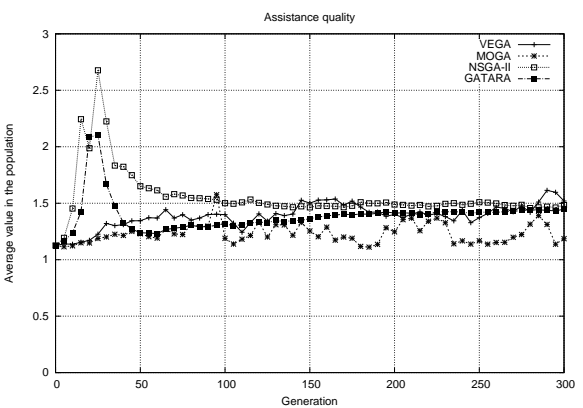

(a)

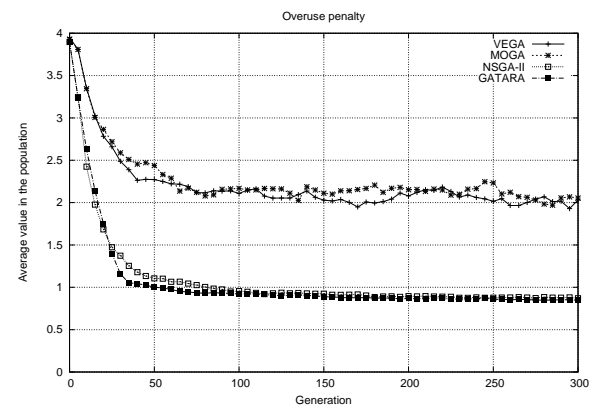

(c)

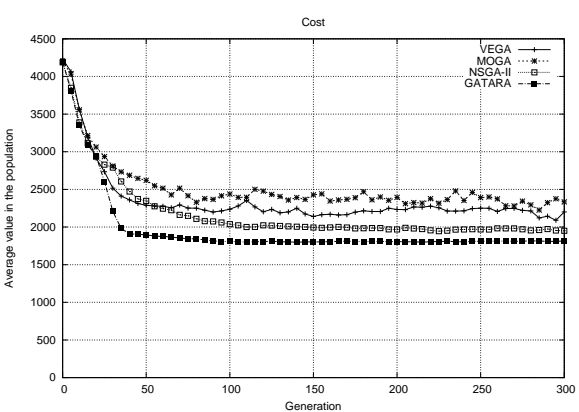

(b)

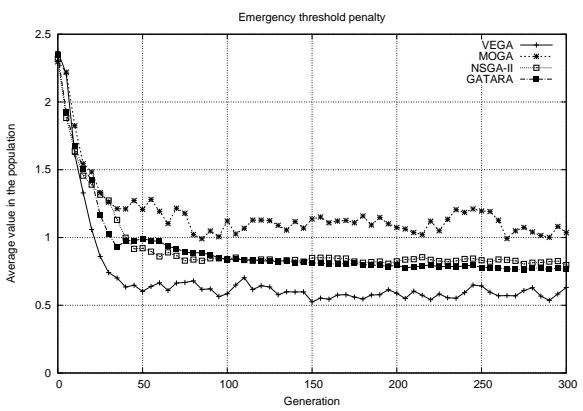

(d)

Figure 6: Evolution of the mean value of the objective functions for the individuals in the population when varying the genetic algorithm in the 10 suppliers scenario. 


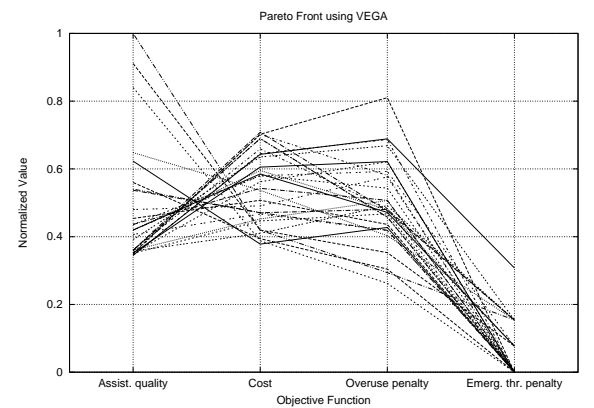

(a)

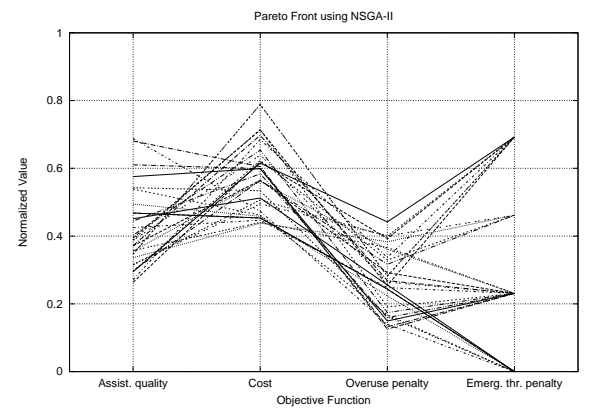

(c)

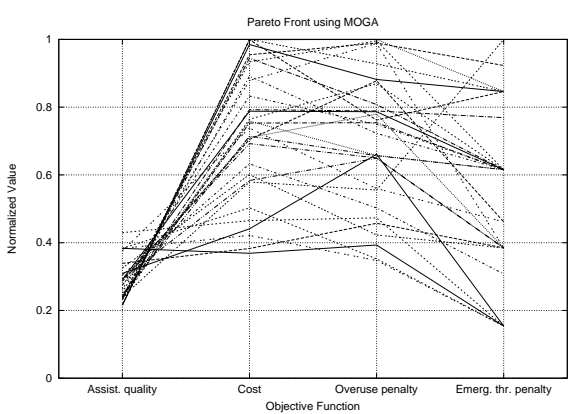

(b)

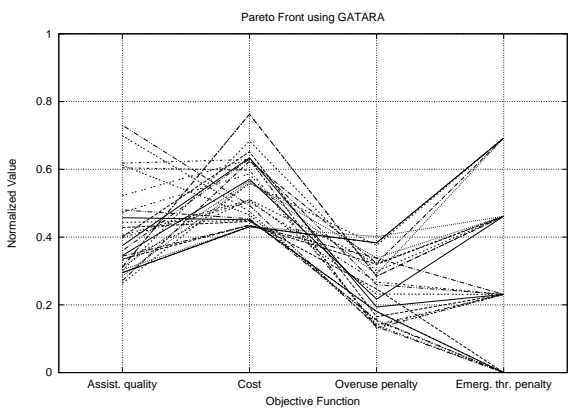

(d)

Figure 7: Pareto front obtained when varying the genetic algorithm in the 10 suppliers scenario: (a) VEGA, (b) MOGA, (c) NSGA-II, and (d) GATARA. 


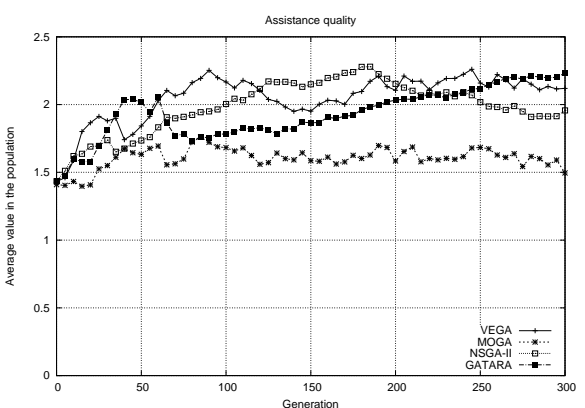

(a)

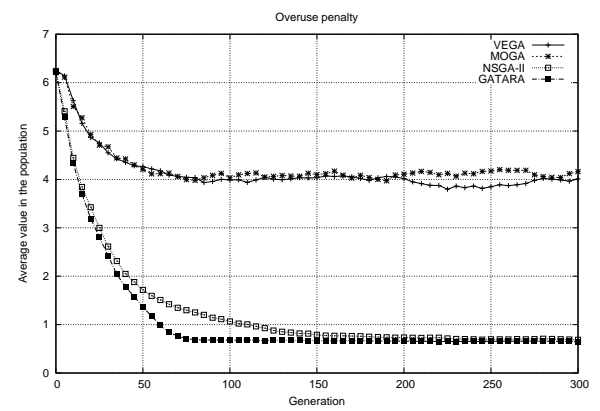

(c)

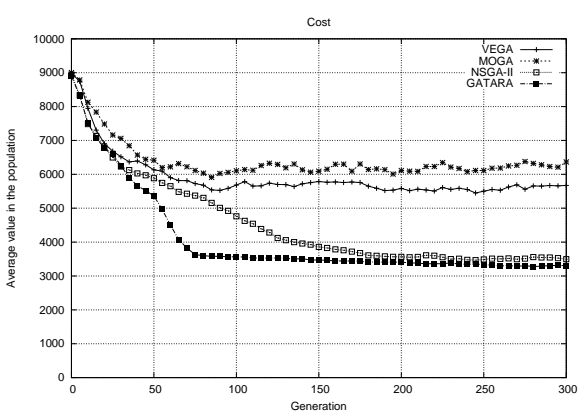

(b)

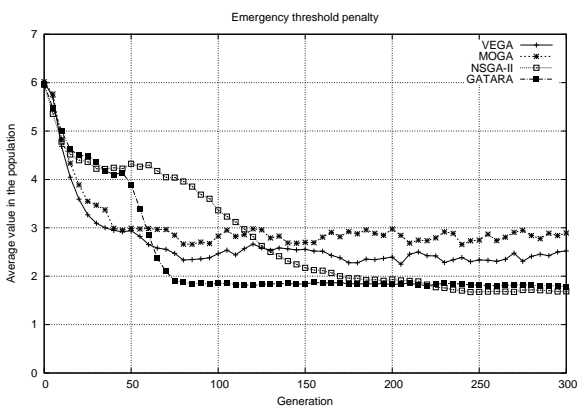

(d)

Figure 8: Evolution of the mean value of the objective functions for the individuals in the population when varying the genetic algorithm in the 20 suppliers scenario. 


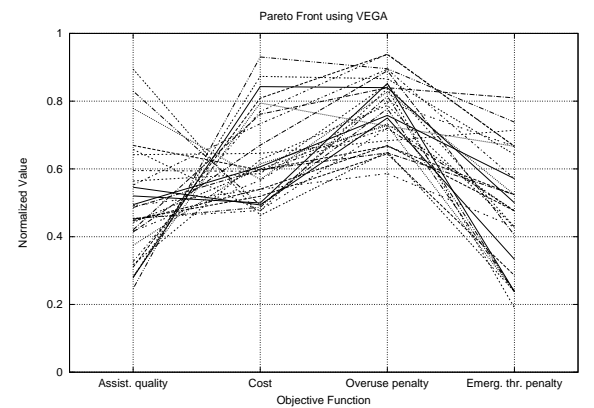

(a)

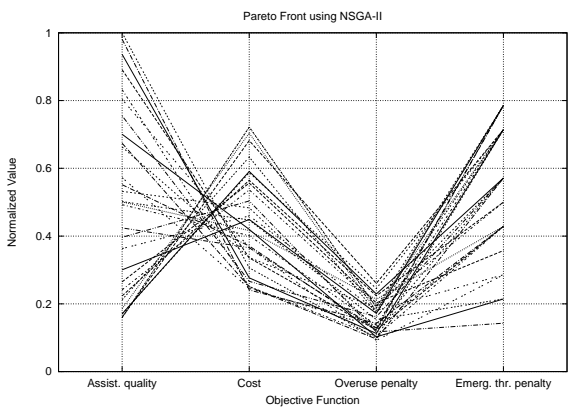

(c)

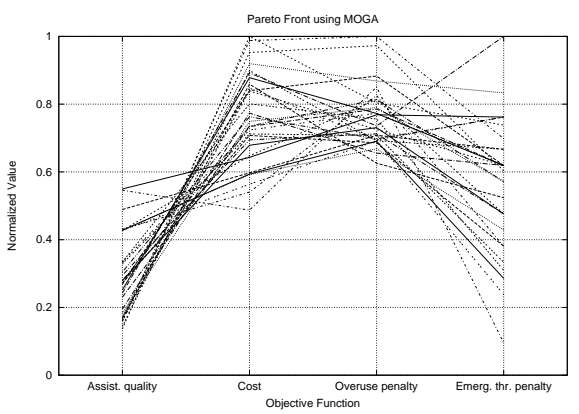

(b)

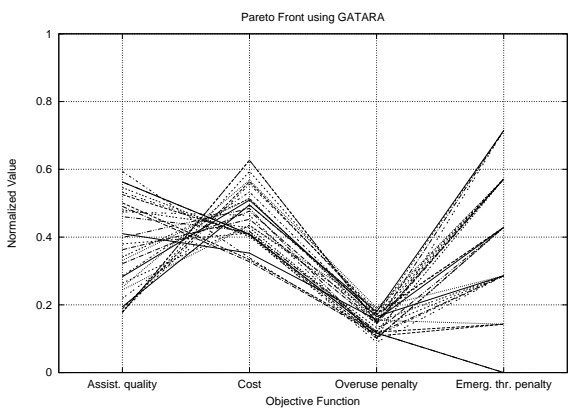

(D)

Figure 9: Pareto front obtained when varying the genetic algorithm in the 20 suppliers scenario: (a) VEGA, (b) MOGA, (c) NSGA-II, and (d) GATARA. 
function, where VEGA is the winner.

The convergence speed of the three schemes is similar, even if GATARA reaches a stabilization point sooner than the rest of the approaches, thus making difficult to select the best algorithm. That is the reason why we study the distribution of the final solutions obtained in the four dimensions after 300 generations, as shown in Figure 7. Among the four algorithms, GATARA and NSGA-II generate fewer extreme values, with a fairly even distribution of solutions in all dimensions. In fact, since GATARA mainly increases the convergence speed of the NSGA-II algorithm, it is logical to obtain such similarity in the distribution of solutions for both algorithms. VEGA achieves very good results for the emergency threshold penalty, but several of the values for the assistance quality are too high or too close to each other. MOGA is not able to achieve enough variety in the assistance quality, with little differences between the solutions, although there is considerable diversity in the other functions. Our proposal is the most appropriate algorithm to obtain a variety of solutions with acceptable quality, allowing decisions to be flexible enough to adapt to different situations and priorities.

Since the behavior of the algorithms may vary in different environments, we studied how the results are affected by using the scenario with 20 suppliers. As shown in Figure 8, GATARA clearly outperforms the rest of schemes after 300 generations for all the functions, except the assistance quality penalty, where all the algorithms behave similarly. The convergence speeds of the four schemes is similar during the first 50 generations. Surprisingly, NSGA-II presents problems to reach the optimum solutions for the cost and emergency threshold functions, requiring more than 150 generations to stabilize. Again, this behavior indicates that NSGA-II explores the search space during the first few generations to later focus on the best areas detected. The hybridization allows GATARA to overcome this drawback, achieving the best solution set in less than 80 generations.

Finally, Figure 9 represents the distribution of the values for the four objective functions in the 20 suppliers scenario. Both VEGA and MOGA present excessively high values for the cost and overuse functions, while MOGA again 
shows deficiencies in terms of variety of values for the assistance quality function. The values for the assistance quality penalty function are also very high in NSGA-II, whereas GATARA is able to find a set of solutions with values that are reduced and diverse enough for all the penalty functions. Therefore, GATARA is a suitable algorithm to generate approximations to the Pareto front with a high degree of uniformity.

\section{Conclusions}

Intelligent Transportation Systems are beginning to be implemented in vehicles from different manufacturers, as a combination of communication systems between vehicles (V2V), and between vehicles and the external infrastructure (V2I). Using this technology together with Artificial Intelligence (AI) systems can be helpful at reducing the assistance time of the injured people in an accident. In this work we showed how Multi-objective Genetic Algorithms make it possible to generate optimal resource sets automatically when an accident is notified and its severity estimated.

Using the resource model presented, there are different multi-objective algorithms that can be used to select the most appropriate resource set, taking into account four main objectives: maximizing the assistance quality, minimizing cost, minimizing the penalty for overuse of resources, and minimizing the penalty for violation of the emergency threshold. We propose a Genetic Algorithm for Traffic Accidents Resource Allocation (GATARA), based on the existing NSGA-II algorithm, modifying it to include hybridization through memetics to increase the convergence speed towards the Pareto front. GATARA still has the advantages of the original NSGA-II approach with respect to other algorithms, such as VEGA and MOGA, since our proposal does not give preference to one function over others, and it is very efficient at obtaining a uniform distribution for solutions using the different objective functions. However, the memetic version of NSGA-II allows for a faster search by improving the nondominated suboptimal solutions found during the search process. 
As future work, it would be interesting to study the system for a long time period to assess its behavior once deployed in a real environment. The simulation would include the modeling of traffic accidents in terms of their frequency and other factors, such as vehicles involved, average number of occupants, etc.

\section{Acknowledgments}

This work was partially supported by the Ministerio de Ciencia e Innovación, Spain, under Grant TIN2011-27543-C03-01, and by the Diputación General de Aragón, under Grant "subvenciones destinadas a la formación y contratación de personal investigador".

\section{References}

Affenzeller, M., Wagner, S., Winkler, S., 2007. Self-adaptive Population Size Adjustment for Genetic Algorithms. In: Moreno Daz, R., Pichler, F., Quesada Arencibia, A. (Eds.), Computer Aided Systems Theory EUROCAST 2007. Vol. 4739 of Lecture Notes in Computer Science. Springer Berlin / Heidelberg, pp. 820-828.

Amcoex, Inc., 2012. Cost of use of sanitary vehicles provided by Amcoex Ambulances, Spain. Available at http://www.amcoex.es/WebAmbulancias/tarifas.htm.

Bäck, J. H., 1996. Evolutionary Algorithms in Theory and Practice. Springer, Oxford.

Corne, D. W., Jerram, N. R., Knowles, J. D., Oates, M. J., 2001. PESA-II: region-based selection in evolutionary multiobjective optimization. In: Proceedings of the genetic and evolutionary computation conference (GECCO2001).

Corne, D. W., Knowles, J. D., Oates, M. J., 2000. The Pareto envelope-based selection algorithm for multiobjective optimization. In: Proceedings of the 
sixth international conference on parallel problem solving from Nature. pp. $18-20$.

Dawkins, R., 1976. The Selfish Gene. Oxford University Press, UK.

Deb, K., Goel, T., 2001. A hybrid multi-objective evolutionary approach to engineering shape design. In: Proceedings of the first international conference evolutionary multi-criterion optimization, EMO 2001.

Deb, K., Pratap, A., Agarwal, S., Meyarivan, T., 2002. A fast and elitist multiobjective genetic algorithm: NSGA-II. IEEE Transactions on Evolutionary Computation 6 (2), 182-197.

Dirección General de Tráfico (DGT), 2010. The main statistics of road accidents. Spain. Available at http://www.dgt.es/portal/es/seguridad_ vial/estadistica.

Eiben, A., Smith, J., 2003. Introduction to evolutionary computing. Springer.

Erfani, T., Utyuzhnikov, S. V., 2010. Directed search domain: A method for even generation of pareto frontier in multiobjective optimization. Journal of Engineering Optimization 12, 1-18.

Eurostat: Statistical Office of the European Communities, 2012. Transport statistics in the EU. Available at http://epp.eurostat.ec.europa.eu/ portal/page/portal/transport/data/main_tables.

Figueiredo, L., Jesus, I., Machado, J. A. T., Ferreira, J. R., Martins, J. L., 2001. Towards the development of intelligent transportation systems. IEEE Intelligent Transportation Systems 25 (81), 1206-1211.

Fogue, M., Garrido, P., Martinez, F. J., Cano, J.-C., Calafate, C., Manzoni, P., October 2011. Prototyping an Automatic Notification Scheme for Traffic Accidents in Vehicular Networks. In: 4th IFIP Wireless Days Conference. pp. $1-5$. 
Fogue, M., Garrido, P., Martinez, F. J., Cano, J.-C., Calafate, C., Manzoni, P., To be published in September 2012a. A realistic prototype for automatic accident detection and assistance through vehicular networks. IEEE Vehicular Technology Magazine.

Fogue, M., Garrido, P., Martinez, F. J., Cano, J.-C., Calafate, C. T., Manzoni, P., 2012b. Using data mining and vehicular networks to estimate the severity of traffic accidents. In: Casillas, J., Martinez-Lopez, F. J., Corchado, J. M. (Eds.), Management Intelligent Systems. Vol. 171 of Advances in Intelligent Systems and Computing. Springer Berlin Heidelberg, pp. 37-46.

URL http://dx.doi.org/10.1007/978-3-642-30864-2_4

Fonseca, C. M., Fleming, P. J., 1993. Multiobjective genetic algorithms. In: IEEE colloquium on Genetic Algorithms for Control Systems Engineering.

Indraneel, D., Dennis, J. E., 1998. Normal-boundary intersection: A new method for generating the pareto surface in nonlinear multicriteria optimization problems. SIAM Journal on Optimization 8 (3), 631-657.

Jones, D. F., Mirrazavi, S. K., Tamiz, M., 2002. Multiobjective meta-heuristics: an overview of the current state-of-the-art. European Journal of Operational Research 137 (1), 1-9.

Knowles, J. D., Corne, D. W., 2000. M-PAES: a memetic algorithm for multiobjective optimization. In: Proceedings of the 2000 congress on evolutionary computation.

Koljonen, J., Alander, J. T., 2006. Effects of population size and relative elitism on optimization speed and reliability of genetic algorithms. In: Proceedings of the Ninth Scandinavian Conference on Artificial Intelligence (SCAI 2006). pp. 54-60.

Martinez, F., Toh, C.-K., Cano, J.-C., Calafate, C., Manzoni, P., summer 2010. Emergency services in future intelligent transportation systems based on ve- 
hicular communication networks. IEEE Intelligent Transportation Systems Magazine 2 (2), 6-20.

Medynet, 2012. Environment organization in accidents with multiple injured passengers. Available at http://www.medynet.com/usuarios/ jraguilar/Organizacion $\% 20$ del $\% 20$ entorno $\% 20$ en $\% 20$ incidentes $\% 20$ con $\%$ 20multiples\%20victimas. \%20Seguridad.pdf.

Messac, A., Ismail-Yahaya, A., Mattson, C. A., 2003. The normalized normal constraint method for generating the pareto frontier. Structural and multidisciplinary optimization 25 (2), 86-98.

Messac, A., Mattson, C., 2004. Normal constraint method with guarantee of even representation of complete pareto frontier. American Institute of Aeronautics and Astronautics Journal 42 (10), 2101-2111.

Miller, J., june 2008. Vehicle-to-vehicle-to-infrastructure (V2V2I) intelligent transportation system architecture. In: 2008 IEEE Intelligent Vehicles Symposium. pp. $715-720$.

Moscato, P., 1989. On Evolution, Search, Optimization, Genetic Algorithms and Martial Arts - Towards Memetic Algorithms. Tech. rep., Caltech Concurrent Computation Program 158-79, California Institute of Technology. Pasadena, CA, USA.

National Highway Traffic Safety Administration (NHTSA), 2012. FTP Site for the General Estimates System (GES). Available at ftp://ftp.nhtsa.dot.gov/GES/.

OpenStreetMap, 2012. A collaborative project to create a free editable map of the world. Available at http://www.openstreetmap.org.

Paquete, L., Stutzle, T., October 2003. A two-phase local search for the biobjective traveling salesman problem. In: Proceedings of the second international conference on evolutionary multi-criterion optimization, EMO 2003. 
Pareto, V., 1909. Cours d'economie politique. Paris.

Schaffer, J. D., July 1985. Multiple objective optimization with vector evaluated genetic algorithms. In: Proceedings of the First International Conference on Genetic Algorithms and Their Applications.

Shukla, P., Deb, K., 2007. On finding multiple pareto-optimal solutions using classical and evolutionary generating method. European Journal of Operational Research 181 (3), 1630-1652.

Spanish Ministry of Health, 1998. Spanish Royal Decree 619/1998 of 17 April establishing the technical features, medical equipment and staff of medical transport vehicles on the road. Available at http://noticias.juridicas. com/base_datos/Admin/rd619-1998.html.

Srinivas, N., Deb, K., 1994. Multiobjective optimization using nondominated sorting in genetic algorithms. Journal Evolutionary Computation 2 (3), 221248.

Teich, J., 2001. Pareto-front exploration with uncertain objectives. In: Zitzler, E., Thiele, L., Deb, K., Coello Coello, C., Corne, D. (Eds.), Evolutionary Multi-Criterion Optimization. Vol. 1993 of Lecture Notes in Computer Science. Springer Berlin / Heidelberg, pp. 314-328.

Utyuzhnikov, S., Fantini, P., Guenov, M., 2009. A method for generating a well-distributed pareto set in nonlinear multiobjective optimization. Journal of Computational and Applied Mathematics 223 (2), 820-841.

Zitzler, E., Deb, K., Thiele, L., 2000. Comparison of multiobjective evolutionary algorithms: empirical results. IEEE Transactions on Evolutionary Computing 8 (2), 173-195. 